\title{
Multi-gas sensing based on photoacoustic spectroscopy using tunable laser diodes
}

\author{
Jean-Philippe Besson*, Stéphane Schilt, Luc Thévenaz \\ Laboratory of Nanophotonics and Metrology (NAM), Swiss Federal Institute of Technology (EPFL), CH-1015 Lausanne, Switzerland
}

Received 8 October 2003; accepted 12 November 2003

\begin{abstract}
Multi-hydrogenated compounds detection based on photoacoustic (PA) spectroscopy is reported. Three near-infrared semiconductor lasers are used with a resonant PA cell operated in its first longitudinal mode to monitor methane, water vapour and hydrogen chloride in the parts per million range. The design of our cell results from simulations performed in order to optimise its performances. Influence of the buffer gas on the PA signal has also been analysed, both theoretically and experimentally. A reduction of the PA signal of almost one order of magnitude has been observed between $\mathrm{N}_{2}$ and He, which demonstrates the importance of the buffer gas in PA spectroscopy. Finally, detection limits of $0.5 \mathrm{ppm}$ of $\mathrm{CH}_{4}$ and $3 \mathrm{ppm}$ of $\mathrm{HCl}$ has been achieved experimentally in nitrogen and an $\mathrm{H}_{2} \mathrm{O}$ sensitivity of 0.2 ppm has been estimated.

(c) 2004 Elsevier B.V. All rights reserved.
\end{abstract}

Keywords: Photoacoustic spectroscopy; Multi-gas sensing; Trace gas monitoring; DFB lasers

\section{Introduction}

The interest for compact and reliable trace gas sensors has considerably increased in recent years. A precise monitoring of a large variety of species in different gas mixtures and at various concentration levels, ranging from parts per billion (ppb) to several hundreds of parts per million ( $\mathrm{ppm})$, is required in various fields of applications. Relevant domains include, for example, atmospheric researches [1], combustion processes [2], medical diagnostic by breath analysis [3], food industry [4] or pollution monitoring [5]. Industrial process control is also of great importance, as extremely high purity gas mixtures are usually employed and level of impurities or contaminants must be precisely controlled at trace levels. Several types of impurities need to be monitored, such as ammonia and acids ( $\mathrm{HF}, \mathrm{HCl}, \mathrm{HBr}$ ) in semiconductor clean room applications or moisture contamination in the manufacturing of electronics or optical devices, such as laser diodes [6].

Laser-based spectroscopic techniques are very attractive methods for trace gas monitoring, as they have demonstrated simultaneous high sensitivity and selectivity. Monochro-

* Corresponding author.

E-mail address: jean-philippe.besson@epfl.ch (J.-P. Besson). maticity of laser sources enables to achieve a high selectivity, as a single absorption line of the substance to be analysed can be probed with a laser, avoiding interference signals from other species. A high sensitivity is reached using modulation techniques, such as wavelength modulation spectroscopy (WMS), frequency modulation spectroscopy (FMS) or photoacoustic spectroscopy (PAS). Among these techniques, PAS is very attractive due to its simplicity, high sensitivity and large dynamic range. It measures directly the amount of modulated laser radiation absorbed in the analysed sample and converted into pressure energy via non-radiative relaxation processes. As this technique depends linearly on the absorbed energy, photoacoustic-based systems requiring extreme sensitivities (in the ppb range) have been developed using mid-infrared gas lasers ( $\mathrm{CO}$ or $\mathrm{CO}_{2}$ lasers), which are able to deliver several watts of optical power in a spectral range where many molecules exhibit their strongest fundamental vibrational transitions. However, many applications do not require such extreme sensitivities and in this case, near-infrared distributed-feedback (DFB) laser diodes are of great interest. These semiconductor devices are very compact and reliable light sources operating at room temperature and provide a long lifetime. They are commonly produced in the spectral range of optical fibres telecommunications $(1.3-1.65 \mu \mathrm{m})$, where overtone vibration bands of many molecules of interest occur $\left(\mathrm{CO}_{2}, \mathrm{H}_{2} \mathrm{O}, \mathrm{NH}_{3}, \mathrm{HCl}, \mathrm{CH}_{4}, \mathrm{HF}\right.$, 
$\left.\mathrm{C}_{2} \mathrm{H}_{2}, \ldots\right)$. Although laser diodes have much reduced optical power in comparison to mid-infrared gas lasers (typically a ten of a milliwatt against several watts), properly designed photoacoustic (PA) systems based on DFB laser diodes have the potential to reach ppm or sub-ppm detection limits for many species of interest. The compatibility of near-infrared DFB lasers with standard telecommunications optical fibres is another key advantage of these lasers. The use of optical fibres simplifies the design of a gas sensor, especially in the case of multi-components detection. A simultaneous monitoring of several species with a single instrument usually requires the use of several lasers, as the typical tuning range of a DFB laser is limited to a few nanometres, whereas the separation between absorption bands of different species is generally much higher. Therefore, one laser per substance is usually required. Optical fibres strongly simplify the coupling of the emission of several lasers into a single measurement cell, eliminating the use of expensive transfer optics (lenses, mirrors, beamsplitters). The use of fibres enables also to reduce the dimensions of the system, as fibered components are more compact than bulk elements.

We report in this paper preliminary results on the development of a multi-gas PA instrument. The interest of this system consists in the possibility to detect several species at trace level with a single instrument. In these experiments, we have been interested in the detection of three hydrogenated compounds, methane $\left(\mathrm{CH}_{4}\right)$, water vapour $\left(\mathrm{H}_{2} \mathrm{O}\right)$ and hydrogen chloride $(\mathrm{HCl})$, using three near-infrared DFB lasers and a resonant PA cell. Theoretical background of PAS is first reviewed and considerations about the optimisation of the design of a PA cell adapted to this application are discussed. Then, the realisation of our system is described and experimental results are presented.

\section{Photoacoustic spectroscopy}

\subsection{Theoretical background}

PAS is a calorimetric method in which the optical energy absorbed in a gas sample is directly measured through the heating induced in the medium. It is therefore radically different from conventional spectroscopic techniques based on Beer-Lambert law, in which the energy transmitted through the sample is detected. The conversion from optical energy to heat is induced by molecular absorption of photons of proper wavelength and subsequent non-radiative relaxation of the excited state (collisional relaxation). The small local temperature variation of the sample is accompanied by a pressure variation. When the deposited optical energy is modulated (for example by an intensity or wavelength modulation of the laser), a periodic heating is produced, generating also a modulation of the sample pressure. This constitutes an acoustic wave (a sound), which can be detected by a miniature microphone. The amplitude $S_{\mathrm{PA}}$ of this acoustic wave is directly proportional to the sample heating, so to the incident optical power $P_{0}$ and to the molecular absorption coefficient $\alpha$ :

$S_{\mathrm{PA}}=C_{\mathrm{cell}} \alpha P_{0}$

where $C_{\text {cell }}$ is a proportionality coefficient, called the cell constant, describing the conversion from optical to acoustic energy. This conversion depends not only from the analysed gas, but also from other external parameters, such as the modulation frequency $f$ or the chemical composition of the buffer gas. A $1 / f$ dependence of the PA signal occurs as the energy absorbed in a modulation cycle decreases as the inverse of the modulation frequency. The composition of the buffer gas influences also the conversion from optical energy to heat (and therefore to acoustic energy) through several physical parameters, such as the buffer gas density, molar mass and specific heat.

The theory of the generation and detection of the PA signal in a cylindrical cell has been described in detail by several authors (see for example [7-10]). As the generated sound is very weak, resonant configurations are usually used. In such geometries, the PA cell is designed to constitute an acoustic resonator and the laser is modulated at a resonant frequency of this cavity. The acoustic wave is thus amplified by a resonance effect, the enhancement coefficient being the quality factor $Q_{j}$ of the acoustic resonance. In resonant operation, an acoustic standing wave oscillates in the cavity and the cell constant is given by:

$C_{\text {cell }}=\frac{Q_{j}}{\omega_{j}} \frac{(\gamma-1) I_{j} L_{\mathrm{c}}}{V_{\mathrm{c}}} p_{j}\left(r_{\mathrm{M}}, \omega_{j}\right)$

where $\gamma=C_{\mathrm{p}} / C_{\mathrm{v}}$ is the ratio of the specific heat at constant pressure and constant volume, $\omega_{j}$ the angular resonance frequency, $V_{\mathrm{c}}$ the cell volume, $L_{\mathrm{c}}$ the cell length, $p_{j}\left(r_{\mathrm{M}}, \omega_{j}\right)$ the value of the normalised acoustic mode at the position $r_{\mathrm{M}}$ of the microphone and $I_{j}$ the overlap integral between the laser beam distribution $g(r)$ and the acoustic mode $p_{j}\left(r, \omega_{j}\right)$ of the cavity:

$I_{j}=\frac{1}{L_{\mathrm{c}}} \int_{V} \mathrm{~d} V g(r) p_{j} \times\left(r, \omega_{j}\right)$

The index $j$ describes the acoustic mode of interest. In a cylindrical resonator, three different kinds of modes occur: longitudinal modes (named by an index $k$ ), azymuthal modes (index $m$ ) and radial modes (index $n$ ), so that $j=k, m, n$. A sensitive PA-based spectrometer aims at maximising the amplitude of the generated acoustic wave. This can be accomplished by various means. Of course, a strong absorption line and a high power laser are desirable, but for a given absorption feature and laser, the only way of improving the sensitivity consists in an increase of the cell constant and in a reduction of the background noise. A major challenge of PAS is to determine a proper design in order to reach the highest cell constant as possible. The objective is to optimise the cell geometry, choosing the proper type of resonance and suitable cell dimensions, in order to maximise Eq. (2). Practical considerations must still be taken into account and restrict the choice of the cell dimensions: 
1. Manufacturing constraints prevent the use of too small cells, as it is necessary to place the microphone or the gas inlet and outlet.

2. As the acoustic and electronic noise varies as $1 / f$, the modulation frequency can not be too small. Practically, resonance frequencies lower than approximately $1 \mathrm{kHz}$ should be avoided. The resonant frequencies depend directly on the resonator dimensions:

$\omega_{j}=2 \pi f_{j}=\pi c_{\mathrm{s}} \sqrt{\left(\frac{k}{L_{\mathrm{eff}}}\right)^{2}+\left(\frac{\alpha_{m n}}{R_{\mathrm{c}}}\right)^{2}}$

where $c_{\mathrm{S}}$ is the sound velocity in the medium, coefficients $\alpha_{m n}$ are related to the zeros of the derivatives of Bessel functions and $L_{\mathrm{eff}}$ the effective length of the resonator. This parameter differs from the geometrical length $L_{\mathrm{c}}$ by a correction factor due to boundary effects at the resonator ends [11]:

$$
L_{\mathrm{eff}}=L_{\mathrm{c}}+\frac{16}{3 \pi} R_{\mathrm{c}}
$$

3. In particular experimental conditions, some geometries are more advantageous. For example, when high power lasers (such as $\mathrm{CO}_{2}$ lasers) or high flow rates are used, PA cells operated on a radial resonance offer several advantages, as window noise and flow noise can be reduced by proper cell design [12]. However, this is not at all an issue when semiconductor lasers and moderate flow rates are used. Semiconductor lasers have small output power $\left(P_{0}<100 \mathrm{~mW}\right)$, so that the window noise does not limit the sensitivity. For semiconductor lasers-based PA spectrometer, longitudinal resonances offer the best potential to reach a high cell constant.

Next section deals with a theoretical study of the optimisation of a PA cell operated in its first longitudinal mode. The value of the cell constant is simulated as a function of the resonator dimensions $R_{\mathrm{c}}$ and $L_{\mathrm{c}}$, in order to determine the best geometrical configuration.

\subsection{Photoacoustic cell optimisation}

The cell constant given by Eq. (2) depends on several parameters: the cell dimensions, the resonance frequency, the quality factor of the resonance, the laser beam geometry (through the overlap integral $I_{j}$ ) and the position $r_{M}$ of the microphone. However, some of these parameters are not independent, so that the most important in the design of the PA cell are the resonator dimensions $L_{\mathrm{c}}$ and $R_{\mathrm{c}}$. The resonance frequency is directly linked to these parameters through Eq. (4), whereas the quality factor also depends on the cell dimensions and resonance frequency. In fact, the quality factor describes the losses in the acoustic wave propagation, which have different origins. The most important contribution comes from interactions of the wave with the cell walls, leading to viscous and thermal losses $[13,14]$.
These losses are characterised by the so-called viscous and thermal boundary layers [15]:

$d_{\mathrm{visc}, j}=\sqrt{\frac{2 \eta}{\rho_{0} \omega_{j}}}, \quad d_{\mathrm{th}, j}=\sqrt{\frac{2 K M}{\rho_{0} \omega_{j} C_{\mathrm{p}}}}$

where $\rho_{0}$ is the gas density, $\eta$ its viscosity, $K$ its thermal conductivity, $C_{\mathrm{p}}$ its specific heat at constant pressure and $M$ the molar mass. The contribution of the surface losses to the quality factor is given in the case of a longitudinal resonance by [14]:

$Q_{\text {surf }}=\frac{R_{\mathrm{c}}}{d_{\mathrm{visc}, j}+(\gamma-1) d_{\mathrm{th}, j}\left(1+2 R_{\mathrm{c}} / L_{\mathrm{c}}\right)}$

In addition to these surface losses, volume losses also occur $[13,14]$. Their contribution to the quality factor is given by $[15]$ :

$\frac{1}{Q_{\mathrm{vol}}}=\frac{\omega}{2 c_{\mathrm{s}}^{2}}\left[\frac{4}{3} \frac{\eta}{\rho_{0}}+(\gamma-1) \frac{K}{\rho_{0} C_{\mathrm{p}}}\right]$

The total quality factor is given by:

$\frac{1}{Q}=\frac{1}{Q_{\text {surf }}}+\frac{1}{Q_{\text {vol }}}$

but in PA cells operating at pressure higher than $0.1 \mathrm{~atm}$, the surface losses are strongly dominant [16], so that the quality factor is approximated by $Q \cong Q_{\text {surf }}$.

Fig. 1 shows the calculated values of the cell constant and resonance frequency as a function of the resonator dimensions. These simulations are based in Eqs. (2)-(7) and consider optimal laser beam excitation and microphone position, in order to maximise the parameters $I_{j}$ and $p_{j}\left(r_{\mathrm{M}}, \omega_{j}\right)$. For the first longitudinal resonance, this corresponds to a laser beam aligned on the cell axis and the microphone placed in the centre of the resonator. It appears from Fig. 1 that long tubes of small radius give rise to the best performances. Taking into account the conditions from (i) to (iii) given in Section 2.1, we have restricted to cell dimensions $L_{\mathrm{c}} \leq$ $170 \mathrm{~mm}$ and $R_{\mathrm{c}} \geq 3 \mathrm{~mm}$ for the realisation of our PA cell. Best performances are thus expected with $L_{\mathrm{c}}=170 \mathrm{~mm}$ and $R_{\mathrm{c}}=3 \mathrm{~mm}$. They correspond to a theoretical cell constant $C_{\text {cell }}=8615 \mathrm{~Pa} \mathrm{~cm} \mathrm{~W}^{-1}$ and a resonance frequency $C_{\text {cell }}=1002 \mathrm{~Hz}$ in nitrogen.

\section{Experimental}

\subsection{Apparatus}

Our experimental set-up consists of three lasers, a resonant PA cell and a detection system. The apparatus is shown schematically in Fig. 2. Two telecommunications DFB laser diodes were used for the detection of methane around $1651 \mathrm{~nm}$ and water vapour around $1369 \mathrm{~nm}$. These lasers are fibre-coupled and the optical fibre ends with a beam collimator directly mounted on the face of the first 

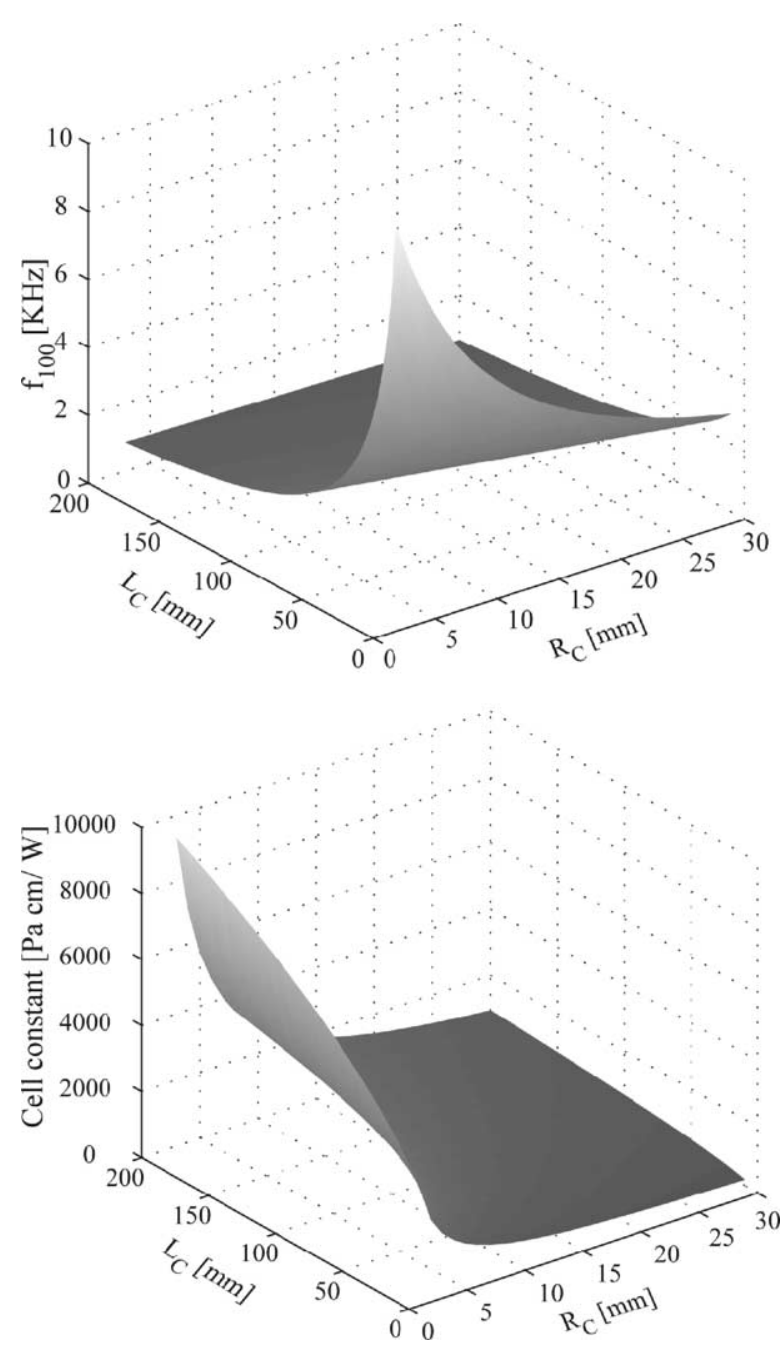

Fig. 1. Theoretical dependence of the cell constant and resonance frequency of the first longitudinal mode [ 1000$]$ of a cylindrical resonator as a function of the cell dimensions (radius $R_{\mathrm{c}}$ and length $L_{\mathrm{c}}$ ).

buffer volume of the PA cell, which facilitates the light coupling into the cell. The detection of hydrogen chloride around $1742 \mathrm{~nm}$ was made at this time with a non-fibered DFB laser used in a free-space configuration. The laser was mounted on micrometer linear stages in order to enable a precise alignment of the beam into the PA cell. In addition, a collimating lens of short focal length and numerical aper- ture of 0.5 was used to collimate the diverging laser emission. The laser beam entered the PA cell through the output window. In the future, a fibered laser will also be used for $\mathrm{HCl}$, which will simplify the design of the system.

The temperature of each laser was tuned to reach the appropriate absorption line and the lasers were intensity-modulated, via a modulation of their injection current, at a frequency corresponding to the first longitudinal resonance of the acoustic resonator. The laser wavelength was adjusted using a reference cell containing a high concentration of the species to be detected. The generated sound wave was detected with an electret microphone placed at the centre of the resonator (i.e. at the maximum of the standing acoustic wave). The signal amplitude was measured using a lock-in amplifier, with a time constant usually set to $1 \mathrm{~s}$.

The home-made PA cell is built out of stainless steel. It consists in two buffer volumes and a central cylindrical resonator designed to be operated in its first longitudinal mode. The laser beam goes across the cell on its axis. Each of the buffer volumes was built with a movable piston, enabling to easily adjust its length. The influence of the volumes length on the coupling between the ambient acoustic noise and the resonator was investigated, in order to determine the most favourable geometry. The flexibility of our cell also includes the possibility to exchange the resonator itself. Two resonators of different radius of $R_{\mathrm{c}}=3$ and $6 \mathrm{~mm}$ and of identical length $L_{\mathrm{c}}=170 \mathrm{~mm}$ were built in order to compare the performances of the cells.

PA spectra of methane, water vapour and hydrogen chloride have been measured by tuning the lasers temperature and by recording the generated PA signals. They are shown in Fig. 3 and are compared with the absorption spectra calculated from HITRAN [17]. A good agreement is obtained between PA measurements and calculated spectra. However, a slightly reduced spectral resolution is achieved experimentally, as some weak lines in the methane and water spectra are not completely resolved in the measurements. This is probably due to a shift of the laser wavelength during the current pulses, induced by a heating of the laser. The $\mathrm{HCl}$ experimental spectrum is also fairly noisy, as it has been measured with the less sensitive acoustic resonator, with a low laser power, a weak $\mathrm{HCl}$ concentration and in a rather noisy acoustic environment.

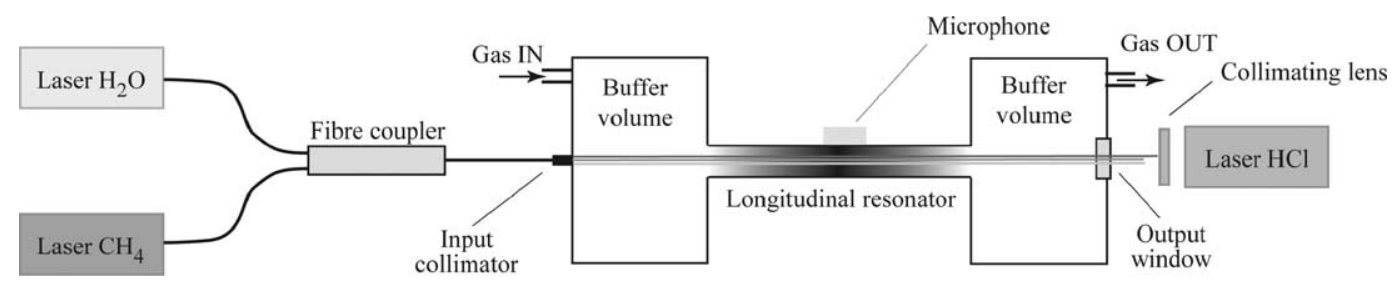

Fig. 2. Schematic representation of our experimental set-up for the measurement of $\mathrm{CH}_{4}, \mathrm{H}_{2} \mathrm{O}$ and $\mathrm{HCl}$ using three near-infrared DFB lasers and a PA cell operated in its first longitudinal resonance. Lasers for $\mathrm{CH}_{4}$ and $\mathrm{H}_{2} \mathrm{O}$ are fibre-coupled and their emission is directly launched into the PA cell using a fibre collimator. Laser for $\mathrm{HCl}$ detection is used in free-space configuration. Its diverging emission is collimated and coupled into the PA cell through the output window. 

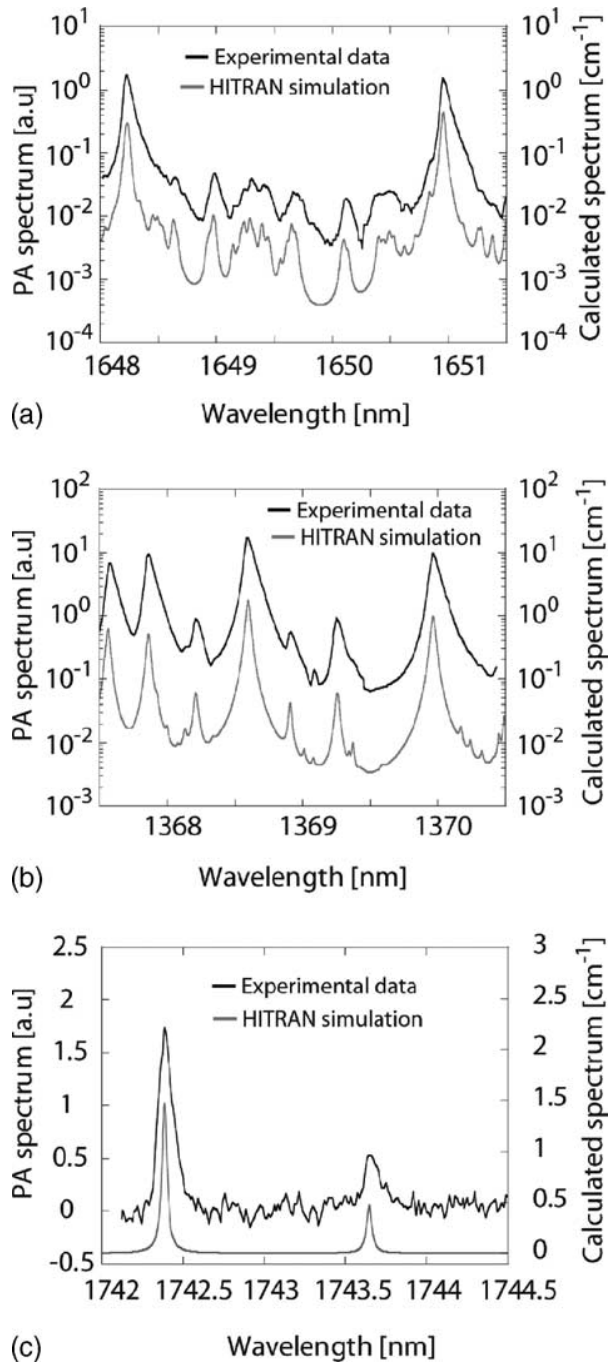

Fig. 3. PA spectra of methane (a), water vapour (b) and hydrogen chloride (c) measured with our experimental set-up. The wavelength was tuned by changing the laser temperature. Black curves are experimental data and grey lines represent the corresponding absorption spectra calculated from HITRAN database.

\subsection{Cell characterisation}

The characterisation of the PA cell was performed by measuring its first longitudinal resonance. A gas mixture of $5000 \mathrm{ppm}$ of methane in nitrogen has been used. The two different resonators of length $L_{\mathrm{c}}=170 \mathrm{~mm}$ and radii $R_{\mathrm{c}}=3$ and $6 \mathrm{~mm}$ have been tested. The measured reso-
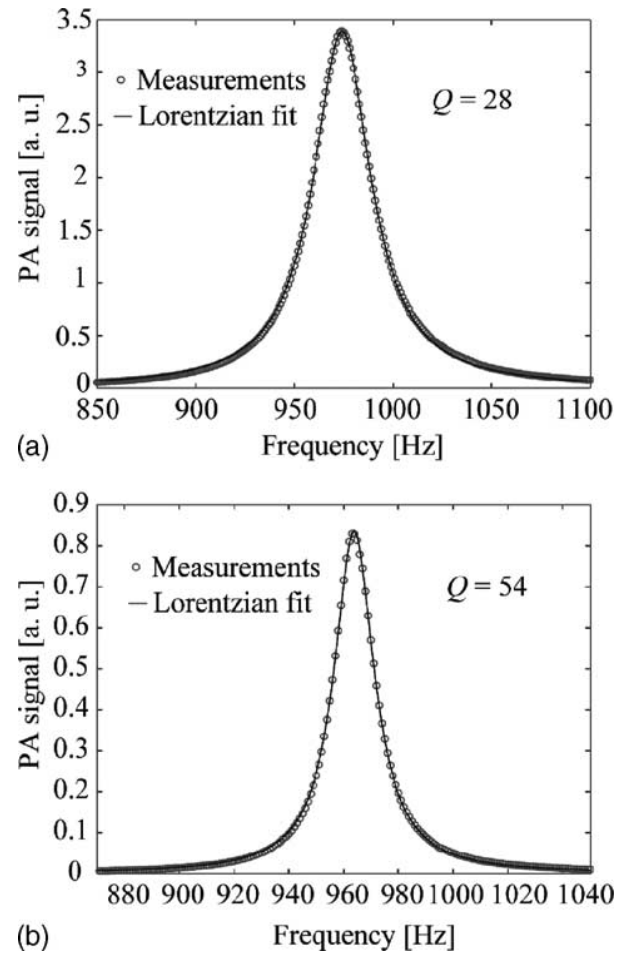

Fig. 4. First longitudinal resonance of our PA cell with two different resonators of length $L_{\mathrm{c}}=170 \mathrm{~mm}$ and radius (a) $R_{\mathrm{c}}=3 \mathrm{~mm}$ and (b) $R_{\mathrm{c}}=6 \mathrm{~mm}$. Circles are experimental points and the curves are the result of a fit by a Lorentzian distribution. The quality factor corresponds to the half width at half maximum of the distribution of the acoustic energy.

nances have been fitted with a Lorentzian distribution in order to extract the quality factor and the resonance frequency of the PA cell. These curves are shown in Fig. 4. A comparison between the measurements and the theoretical values calculated from Eqs. (2), (4) and (7) is summarised in Table 1. A good qualitative agreement is obtained between calculus and measurements. In particular, the experimental quality factors are less than $10 \%$ smaller than the calculated values, which indicates a good quality of the internal surface of our resonators. It can also be seen that the resonance frequencies are situated around $1 \mathrm{kHz}$, which fulfil condition (ii) given in Section 2.1. As expected from the simulations, the smaller radius resonator $\left(R_{\mathrm{c}}=3 \mathrm{~mm}\right)$ has demonstrated the best performances. The experimental cell constant can be derived from Eq. (1) by measuring the PA signal obtained in well-controlled conditions, i.e. with a certified concentration of a gas of known absorption and by

Table 1

Comparison of the performances of the PA cells

\begin{tabular}{|c|c|c|c|c|c|c|c|c|}
\hline \multicolumn{3}{|c|}{ Geometrical parameters } & \multicolumn{3}{|l|}{ Theory } & \multicolumn{3}{|c|}{ Experimental } \\
\hline $\begin{array}{l}\text { Radius } \\
(\mathrm{mm})\end{array}$ & $\begin{array}{l}\text { Length } \\
(\mathrm{mm})\end{array}$ & $\begin{array}{l}\text { Volume } \\
(\mathrm{ml})\end{array}$ & $\begin{array}{l}\text { Frequency } \\
(\mathrm{Hz})\end{array}$ & $Q$ factor & $\begin{array}{l}\text { Cell constant } \\
\left(\mathrm{Pacm} \mathrm{W} \mathrm{W}^{-1}\right)\end{array}$ & $\begin{array}{l}\text { Frequency } \\
(\mathrm{Hz})\end{array}$ & $Q$ factor & $\begin{array}{l}\text { Cell constant } \\
\left(\mathrm{Pa} \mathrm{cm} \mathrm{W}^{-1}\right)\end{array}$ \\
\hline 3 & 170 & 4.8 & 1,002 & 30.1 & 8,615 & 974.2 & 28 & 13,232 \\
\hline 6 & 170 & 19.2 & 973.8 & 58.5 & 4,320 & 963.8 & 54 & 6,552 \\
\hline
\end{tabular}

Theoretical values have been calculated from Eqs. (2), (4) and (7) given in Section 2.1. Experimental values have been measured using a gas mixture of

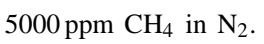


measuring also the laser power $P_{0}$. With the typical sensitivity of $10 \mathrm{mV} \mathrm{Pa}^{-1}$ of our microphone and taking into account the gain $(G=200)$ of its preamplifier, a cell constant of $13,232 \mathrm{~Pa} \mathrm{~cm} \mathrm{~W}^{-1}$ is obtained with the $3 \mathrm{~mm}$ radius resonator and $6552 \mathrm{~Pa} \mathrm{~cm} \mathrm{~W}^{-1}$ with the $6 \mathrm{~mm}$ radius. These values are much higher than the calculated cell constants. This is probably due to an imprecision in the microphone sensitivity used in the experimental determination of the cell constant. The real sensitivity seems to be quite higher than the value of $10 \mathrm{mV} \mathrm{Pa}^{-1}$ given by the manufacturer, which directly affects the experimental value of the cell constant. The experimental cell constant improved by a factor 2 when reducing the resonator radius from 6 to $3 \mathrm{~mm}$, as expected from the calculation.

\subsection{Influence of the buffer gas}

As mentioned in Section 2.1, the PA signal generated in a gas mixture depends not only on the concentration of the analysed species, but also on the buffer gas. In some specific applications, gas traces need to be monitored not only in ambient air, but also in different diluting gases, such as, for example, oxygen $\left(\mathrm{O}_{2}\right)$, helium $(\mathrm{He})$ or nitrogen $\left(\mathrm{N}_{2}\right)$. The composition of the buffer gas influences the cell constant by different effects:

- The resonance frequency is directly proportional to the sound velocity, which depends in particular on the molar mass $M$ of the gas:

$$
c_{\mathrm{s}}=\sqrt{\frac{\gamma R T}{M}}
$$

where $R=8.3144 \mathrm{~J}(\mathrm{~mol} \mathrm{~K})^{-1}$ is the constant of perfect gases and $T$ is the temperature. Resonance frequencies in light molecules gases are much higher than in heavy molecules gases. The inverse behaviour is obtained for the PA signal due to the $1 / f$ dependence of the cell constant.

- Some physical constants are strongly different between two buffer gases. These parameters occur in the expression of the quality factor (density $\rho_{0}$, thermal conductivity $K$, molar mass $M$, specific heat $C_{\mathrm{p}}$, viscosity $\eta$ ), and directly in the cell constant (parameter $\gamma$ ). The values of these parameters are listed in Table 2 for different gases.

- The buffer gas may also influence the shape of the analysed absorption feature, and thus the value of the absorp- tion coefficient on the line centre, as the foreign pressure broadening coefficient can fairly vary between two buffer gases. For example, pressure broadening coefficients of an absorption line of methane measured in the $1.65 \mu \mathrm{m}$ range in different buffer gases have shown a variation as high as $50 \%$ between air and helium [18].

The effect of the buffer gas has been investigated experimentally using our set-up. Gas mixtures made of $100 \mathrm{ppm}$ of $\mathrm{CH}_{4}$ diluted in different buffer gases have been prepared with mass flow controllers. The first longitudinal resonance of our cell has been measured in each diluting gas and a Lorentzian distribution has been fitted on the experimental points in order to extract the resonance frequency, the quality factor and the cell constant. Buffer gases made of various compositions of $\mathrm{N}_{2}$ and $\mathrm{He}$ have been used. Fig. 5 shows the variation of the experimental parameters as a function of the relative concentration of $\mathrm{N}_{2}$ and $\mathrm{He}$. Theoretical values are also represented. These results show that the experimental resonance frequency matches very well the calculate value, excepted in the case of pure helium, where the measurements were more noisy than in other conditions. The variation of the quality factor shows also a clear tendency, which is in good agreement with the calculation. The experimental cell constant presents however a different slope as a function of the $\mathrm{N}_{2} / \mathrm{He}$ mixing ratio than the predicted values. As already discussed in Section 3.2, this is partially due to the imprecise value of the microphone sensitivity used in the determination of the experimental cell constant. However, the reduction of the experimental cell constant measured between pure $\mathrm{N}_{2}$ and pure $\mathrm{He}$ is much higher than expected from theory. The strong difference between $\mathrm{N}_{2}$ and He results mainly from the high difference in the resonance frequencies obtained in pure $\mathrm{N}_{2}$ or $\mathrm{He}$ (respectively around 1 and $2.5 \mathrm{kHz}$ ), but also from the strongly different $(\gamma-1)$ parameter occurring in Eq. (2) of the PA signal. Additional phenomena may still increase the different behaviour observed between $\mathrm{N}_{2}$ and He. The lineshape of the measured absorption feature may fairly differ between these two buffer gases, as previously discussed. A possible frequency-dependent response of the microphone may also contribute to this effect, as the resonance frequency is strongly different between $\mathrm{N}_{2}$ and He. These phenomena may affect the experimental value of the cell constant, but have not been considered in the calculation. All these results confirm that the buffer gas compo-

Table 2

Physical constants of several buffer gases at $20^{\circ} \mathrm{C}$ and $1 \mathrm{~atm}$

\begin{tabular}{lllllll}
\hline Substance & $\rho_{0}\left(\mathrm{~kg} \mathrm{~m}^{-3}\right)$ & $\gamma$ & $M\left(\mathrm{~kg} \mathrm{~mol}^{-1}\right)$ & $\eta\left(\mathrm{Pa} \mathrm{s}^{-1}\right)$ & $K\left(\mathrm{~W}(\mathrm{~m} \mathrm{~K})^{-1}\right)$ & $C_{\mathrm{p}}\left(\mathrm{J}(\mathrm{mol} \mathrm{K})^{-1}\right)$ \\
\hline $\mathrm{He}$ & 0.178 & 1.63 & 0.0040 & $2 \times 10^{-5 \mathrm{a}}$ & $152 \times 10^{-3}$ & 20.9 \\
$\mathrm{~N}_{2}$ & 1.25 & 1.401 & 0.0280 & $1.75 \times 10^{-5}$ & $26 \times 10^{-3 \mathrm{~b}}$ & 29.1 \\
$\mathrm{O}_{2}$ & 1.43 & 1.398 & 0.0320 & $2 \times 10^{-5}$ & $26.7 \times 10^{-3}$ & 29.3 \\
$\mathrm{Air}$ & 1.293 & 1.402 & 0.0288 & $1.8 \times 10^{-5}$ & $25 \times 10^{-3}$ & 29.1 \\
$\mathrm{CO}_{2}$ & 1.98 & 1.293 & 0.0440 & $1.38 \times 10^{-5}$ & $0.71 \times 10^{-3}$ & 36.8 \\
\hline
\end{tabular}

${ }^{\text {a }}$ At $300 \mathrm{~K}$.

b At $25^{\circ} \mathrm{C}$. 


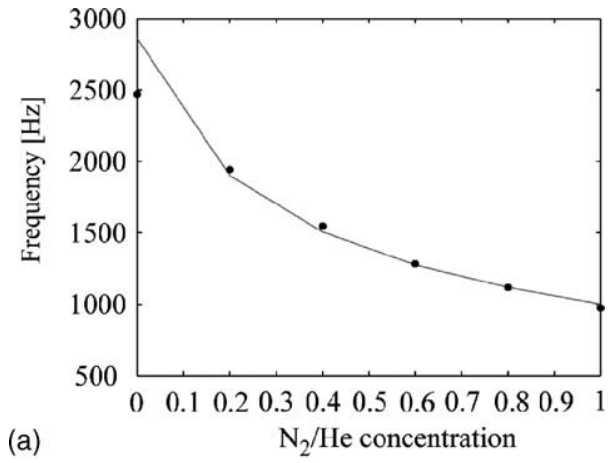

(a)
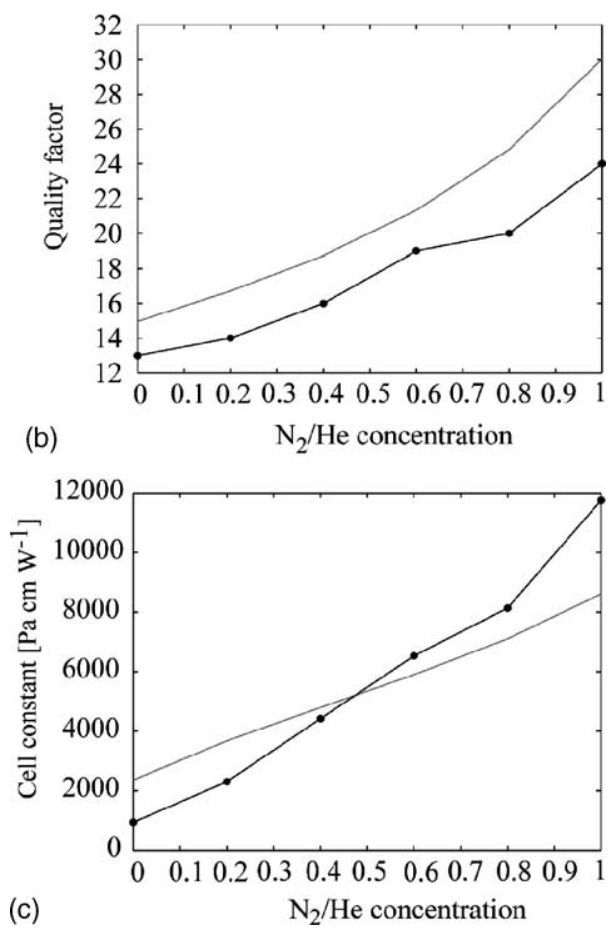

Fig. 5. Variation of the resonance frequency (a), quality factor (b) and cell constant (c) as a function of the buffer gas composition. Circles are experimental data and grey lines are theoretical values calculated from Eqs. (2), (4) and (7) given in Section 2.1

sition is a significant parameter in trace gas monitoring by PAS.

\subsection{Trace gas detection}

An essential parameter for the detection of gas traces is the sensitivity achieved by the system. This sensitivity can be improved by increasing the laser power or by reducing the background noise. In our actual set-up, the predominant noise sources are ambient acoustic noise and microphone intrinsic noise. The flow rate of the gas in the cell is also a critical parameter that must be carefully adjusted. In the present configuration, the maximum flow rate that does not produce any additional noise is limited to $200 \mathrm{sccm}$, due to the small diameter of the resonator. The relatively low power of the semiconductor lasers makes the system insensitive to windows noise. The actual noise measured when flowing

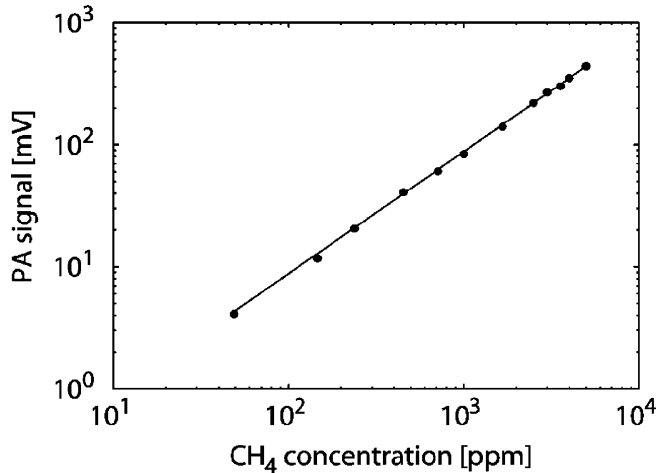

Fig. 6. Response of the PA signal for varying $\mathrm{CH}_{4}$ concentration. The laser emission was centred on the strongest $\mathrm{CH}_{4}$ absorption line at $1651 \mathrm{~nm}$ and the methane concentration was changed by dilution in pure nitrogen using mass flow controllers. The smaller radius resonator $\left(R_{\mathrm{c}}=3 \mathrm{~mm}\right)$ was used.

pure nitrogen through the cell, with an integration time of $1 \mathrm{~s}$ and a preamplifier gain $G=200$ is about $30 \mu \mathrm{V}$. The response of the system for various $\mathrm{CH}_{4}$ concentrations in $\mathrm{N}_{2}$ is illustrated in Fig. 6 and shows a good linearity. From the noise level, a sensitivity of $0.5 \mathrm{ppm}$ (corresponding to a signal-to-noise ratio $\mathrm{SNR}=1$ ) is obtained for $\mathrm{CH}_{4}$ detection in nitrogen with an average laser power of $10 \mathrm{~mW}$ and using the small $R_{\mathrm{c}}=3 \mathrm{~mm}$ resonator. The detection limit of $\mathrm{CH}_{4}$ in pure helium is reduced to $2 \mathrm{ppm}$, due to the much smaller cell constant (see Fig. 5). Present sensitivity for $\mathrm{HCl}$ in nitrogen is only $3 \mathrm{ppm}$, due to a lower laser power around $2 \mathrm{~mW}$. Detection limit for $\mathrm{H}_{2} \mathrm{O}$ has not been measured at this time. However, a value of approximately $0.2 \mathrm{ppm}$ of $\mathrm{H}_{2} \mathrm{O}$ in $\mathrm{N}_{2}$ is estimated from the sensitivity obtained for $\mathrm{CH}_{4}$ and taking into account the ratio of the absorption coefficients of the corresponding absorption lines and the different laser power.

\section{Conclusion}

Multi-hydrogenated compounds detection has been performed by resonant PAS. The reported system is based on a PA cell operated in its first longitudinal mode and three DFB lasers for the detection of $\mathrm{CH}_{4}$ at $1651 \mathrm{~nm}, \mathrm{H}_{2} \mathrm{O}$ at $1369 \mathrm{~nm}$ and $\mathrm{HCl}$ at $1742 \mathrm{~nm}$. The performances of the PA cell have been simulated as a function of the resonator dimensions, in order to determine the more suitable geometry. A PA cell has been built, based on the results of these calculations. A flexible configuration has been chosen, enabling to easily change some of its dimensions. The cell is made of a central cylindrical resonator and two buffer volumes. The length of these volumes is adjustable and two resonators of different radius have been used. The measured cell performances were in good agreement with the calculated values, mainly for the resonance frequency and for the quality factor.

The effect of the buffer gas composition on the PA signal has also been analysed, both theoretically and experimen- 
tally. Measurements made with different $\mathrm{N}_{2} / \mathrm{He}$ mixtures as buffer gas have shown that the PA signal produced by a given concentration of methane changed by almost one order of magnitude when diluted in pure nitrogen or pure helium. This effect is mainly due to the high difference in the resonance frequencies corresponding to these diluting gases, but also to strong differences in the values of some physical constants, such as the $\gamma$ parameter, the gas density or thermal conductivity. Influence of the buffer gas on the measured absorption profile and frequency dependence of the microphone may also contribute to this effect. These results have shown that the buffer gas composition is a significant parameter in trace gas monitoring by PAS.

A detection limit of $0.5 \mathrm{ppm}$ of methane has been achieved in pure nitrogen using a fibre-coupled DFB laser with an average laser power of $10 \mathrm{~mW}$ and using a small $3 \mathrm{~mm}$ radius resonator. The use of optical fibres has enabled to launch the laser emission directly into the PA cell with a fibre collimator, thus simplifying the system design. $\mathrm{CH}_{4}$ sensitivity in pure helium was strongly reduced in comparison to nitrogen and reached only $2 \mathrm{ppm}$. Sensitivity for $\mathrm{HCl}$ was a little worse, due to the lower optical power injected into the PA. A detection limit of only $3 \mathrm{ppm}$ of $\mathrm{HCl}$ in nitrogen was reached in the present configuration. Detection limit for $\mathrm{H}_{2} \mathrm{O}$ has not been measured at this time, but a value of approximately $0.2 \mathrm{ppm}$ of $\mathrm{H}_{2} \mathrm{O}$ in nitrogen has been estimated from the sensitivity obtained for $\mathrm{CH}_{4}$. A reduction of the residual background noise is expected in the future by proper acoustic isolation, and a sub-ppm detection limit for the three hydrogenated compounds (in nitrogen) is expected.

\section{References}

[1] G. Durry, Spectrochim. Acta A 57 (2001) 1855-1863.

[2] R.M. Mihalcea, D.S. Baer, R.K. Hanson, Proc. Combust. Inst. 27 (1998) 95-101.

[3] L. Menzel, A.A. Kosterev, F.K. Tittel, C. Gmachl, F. Capasso, D.L. Sivco, J.N. Baillargeon, A.L. Hutchinson, A.Y. Cho, W. Urban, Appl. Phys. B 72 (2001) 859-863.

[4] H.S.M. de Vries, M.A.J. Wasano, F.J.M. Harren, E.J. Woltering, H.C.P.M. van der Valk, J. Reuss, Postharvest Biol. Technol. 8 (1996) $1-10$.

[5] D.D. Nelson, M.S. Zahniser, J.B. McManus, C.E. Kolb, J.L. Jiménez, Appl. Phys. B 67 (1998) 433-441.

[6] S.-Q. Wu, J.-I. Morishita, H. Masusaki, T. Kimishima, Anal. Chem. 70 (1998) 3315-3321.

[7] L.B. Kreuzer, in: Y.-H. Pao (Ed.), Optoacoustic Spectroscopy and Detection, Academic Press, New York, 1977, pp. 1-25 (Chapter 1).

[8] A. Miklos, P. Hess, Z. Bozoki, Rev. Sci. Instrum. 72 (4) (2001) 1937-1955.

[9] S. Schilt, Détection de traces de gaz à l'aide de lasers à semi-conducteurs, Ph.D. dissertation no. 2525, Swiss Federal Institute of Technology, Lausanne, 2002.

[10] P.L. Meyer, M.W. Sigrist, Rev. Sci. Instrum. 61 (7) (1990) 1779 1807.

[11] L.E. Kinsler, A.R. Frey, A.B. Coppens, J.V. Sanders, Fundamentals of Acoustics, Wiley, New York, 2001.

[12] R. Gerlach, N.M. Amer, Appl. Phys. 23 (1980) 319-326.

[13] R.D. Kamm, J. Appl. Phys. 47 (8) (1976) 3550-3558.

[14] A. Karbach, P. Hess, J. Chem. Phys. 83 (1) (1985) 1075-1084.

[15] P.M. Morse, K.U. Ingard, Theoretical Acoustics, McGraw-Hill, New York, 1968.

[16] L.S. Rothman, et al., J. Quant. Spectrosc. Radiat. Transfer. 60 (1998) 665-710.

[17] C.F. Dewey, in: Y.-H. Pao (Ed.), Optoacoustic Spectroscopy and Detection, Academic Press, New York, 1977, pp. 47-77 (Chapter 3).

[18] V. Zéninari, B. Parvitte, D. Courtois, V.A. Kapitanov, Y.N. Ponomarev, Appl. Phys. B 72 (8) (2001) 953-959. 